FORMATION Formation emploi

Revue française de sciences sociales

128 | octobre-décembre 2014

Pêle-Mêle

\title{
Présentation de l'ouvrage de J. Rose, Mission insertion. Un défi pour les universités
}

Fabienne Maillard

\section{(2) OpenEdition}

1 Journals

Édition électronique

URL : http://journals.openedition.org/formationemploi/4330

DOI : 10.4000/formationemploi.4330

ISSN : 2107-0946

Éditeur

La Documentation française

Édition imprimée

Date de publication : 31 décembre 2014

Pagination : 109-114

ISSN : 0759-6340

\section{Référence électronique}

Fabienne Maillard, «Présentation de l'ouvrage de J. Rose, Mission insertion. Un défi pour les universités », Formation emploi [En ligne], 128 | octobre-décembre 2014, mis en ligne le 20 janvier 2015, consulté le 30 octobre 2020. URL : http://journals.openedition.org/formationemploi/4330 ; DOI : https:// doi.org/10.4000/formationemploi.4330

(c) Tous droits réservés 


\title{
Note de lecture
}

\section{Présentation de l'ouvrage de J. Rose, Mission insertion, un défi pour les universités}

\author{
par Fabienne Maillard,
} sociologue, professeur des universités, université Lille 3

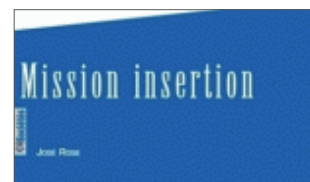

In defi pont les minersites
L'insertion professionnelle à l'issue de l'enseignement supérieur est, depuis plusieurs années, une préoccupation de la puissance publique, qui s'est attachée à réformer les diplômes, les cursus de formation... et même les universités, pour améliorer les relations entre formations et emplois.

Dans cet esprit, une nouvelle mission d'aide à l'insertion professionnelle des étudiants a été confiée aux universités, par la loi dite "LRU » (loi relative aux libertés et responsabilités des universités), adoptée en 2007.

C'est à cette mission que s'intéresse José Rose dans un ouvrage dense qui veut faire le tour de la question. Si l'enjeu est à ses yeux politique et social (on pourrait ajouter économique), il constitue également un enjeu de recherche en raison des questions théoriques et méthodologiques qui ne manquent pas d'être soulevées. Chercheur spécialiste du sujet et directeur scientifique du Céreq (Centre d'études et de recherche sur l'emploi et les qualifications) pendant plusieurs années, José Rose mobilise, pour cette entreprise, la connaissance qu'il a accumulée sur le fonctionnement des relations formations-emplois, mais aussi son expérience d'enseignant-chercheur impliqué dans la mise en place d'un BAIP (bureau d'aide à l'insertion professionnelle) à l'université de Provence. Il le précise d'emblée, c'est dans "une logique de l'action et de la transformation sociale" qu'il inscrit son travail, "avec le souci du service public et une posture de chercheur" (p. 10). Le projet est donc ambitieux et conduit avec passion, au fil de six chapitres qui reviennent méthodiquement sur les obstacles et les questions que posent l'orientation des étudiants et la mobilisation des moyens susceptibles de les aider à accéder plus facilement à l'emploi. 
Le premier chapitre revient sur l'émergence de cette « nouvelle » mission dévolue aux universités, sachant, rappelle l'auteur, que la question des débouchés des sortants de l'enseignement supérieur se pose dès les années 1970.

Pour José Rose, une telle mission est d'autant plus nécessaire qu'elle a l'avantage de répondre "à une attente des étudiants et de la société " (p. 13) ; c'est pourquoi il met en cause l'ambivalence des universitaires à son égard ainsi que l'hétérogénéité des pratiques des universités. Imposée par la loi LRU d'août 2007, la mission d'aide à l'insertion est en effet portée par des structures différentes et changeantes et est inégalement considérée et outillée, ce qui ne favorise ni sa légitimité ni son efficacité.

Or, pour l'auteur, ce projet représente une opportunité de faire évoluer les pratiques universitaires et réduire in fine les inégalités sociales d'accès à l'information, à l'orientation et à l'emploi. À l'encontre d'une certaine diabolisation de la professionnalisation de l'université qui a fait couler beaucoup d'encre ces dernières années, il défend une politique susceptible, selon lui, de favoriser la démocratisation de l'enseignement supérieur. Car ce sont en effet les étudiants les plus démunis qui pâtissent le plus du manque de préparation et d'accompagnement à la vie active.

Alors que nombre d'auteurs s'inquiètent du rapprochement entre les universités et le monde entrepreneurial et dénoncent l'affaiblissement et la marchandisation des savoirs que risquent de produire les réformes de l'enseignement supérieur, José Rose prend le parti inverse, sans négliger de considérer les limites des réformes engagées. Il adopte une position plutôt hétérodoxe, qu'il assume clairement et avec tout autant de vigueur que de rigueur. Il y va pour lui de l'intérêt des étudiants et des universités.

La question de la nécessité d'une telle mission n'est pas pour autant résolue, et l'on aurait apprécié que l'auteur discute les ouvrages critiques publiés dans la dernière décennie. Née avec la première vague (relative) de massification de l'enseignement supérieur, dans le cadre de la première explosion scolaire dans années 1960, la question de l'accès à l'emploi des diplômés du supérieur est depuis récurrente. Tient-elle à des difficultés réelles d'insertion, mais lesquelles et depuis quand ? L'expression même de cette question, face à des modalités d'insertion qui apparaissent rétrospectivement plutôt aisées, ne relève-t-elle pas d'une conception très normative des relations entre formation-diplôme et emploi-qualification ?

José Rose souligne, dans ce chapitre, les réticences des enseignants-chercheurs, qu'i i explique par les changements affectant leur métier comme le fonctionnement des établissements.

Il interpelle également sur le risque de voir une telle mission se développer sur un marché lucratif et réclame, pour contrer ce danger, "une véritable politique d'établissement» (p. 31), à même de valoriser le travail requis pour l'accompagnement des étudiants

Si la démonstration est méthodiquement conduite, la faiblesse des moyens alloués aux établissements et les contraintes qui alourdissent l'activité des enseignants-chercheurs apparaissent peu favorables à la conversion des enseignants-chercheurs. 
En revanche, on peut considérer avec l'auteur qu'une meilleure connaissance, de la part des enseignants (et pas seulement du supérieur) comme des étudiants, du système d'emploi, du fonctionnement du marché du travail et des relations formation-emploi, serait bénéfique à l'ensemble des acteurs du système éducatif.

Le deuxième chapitre expose de manière synthétique les résultats les plus saillants relatifs à l'insertion des sortants de l'enseignement supérieur et discute les notions en usage, comme le " déclassement».

Familier des enquêtes et de leur analyse, José Rose livre des éléments-clés de la connaissance scientifique disponible, forte de très nombreux travaux accumulés depuis une quarantaine d'année, principalement grâce aux travaux que mène le Céreq, mais qui reste selon lui encore trop peu mobilisée par les universitaires.

On ne peut que lui donner raison sur ce point. Ce chapitre est très nourri, éclairant, il a une visée d'information et de pédagogie et contredit utilement la somme des idées reçues qui accompagnent tout ce qui a trait à l'insertion des jeunes.

Il présente, à juste titre, les limites de nombre d'enquêtes réalisées par les universités, tout en dénonçant les tentations adéquationnistes particulièrement développées dans les politiques publiques récentes, que ce soit au niveau national ou local. La position de José Rose est sans ambiguïté : viser " une articulation étroite entre formation, emploi et travail s'avère être une illusion» (p. 61). Renforcer la mission d'aide à l'insertion et réorganiser les dispositifs d'enquête en les rendant plus robustes permettrait, selon lui, de déciller les yeux des uns et des autres et de faire ouvre utile à tous.

Le troisième chapitre dresse une liste des ressources disponibles pour construire un projet professionnel et en montre les avantages différentiels. L'importance des outils mobilisables est mise en valeur.

On dispose là d'une sorte de vademecum à l'intention des enseignants et des étudiants, conçu à la fois pour identifier les outils, repérer leurs potentialités et leurs limites, favoriser et étendre leur usage, et mettre en garde contre une mobilisation non réflexive de ces ressources.

Le " projet » occupe une bonne place dans cette présentation, qui n’omet pas de considérer les problèmes que pose l'injonction à ce projet.

Malgré cette distance critique, la forme de liste que prend ce chapitre peut apparaître déconcertante, la volonté de l'auteur de considérer l'ensemble des ressources mobilisables l'incitant à mettre sur le même pied des objets très divers. Il peut être également difficile de partager sa position délibérément favorable, même si son objectif est d'ouvrir le champ des possibles.

C'est ensuite l'expérience de l'emploi et de l'entreprise dont disposent les étudiants qui occupe le quatrième chapitre. Soucieux de combattre les préjugés trop souvent répandus 
sur le manque d'expérience des jeunes sortant du système éducatif, José Rose rappelle l'importance et la fréquence des expériences de travail acquises par les étudiants tout au long de leur parcours d'études.

Cette fréquence invite à ses yeux à discuter la notion de " débutants " qui les désigne, dès lors qu'ils sont de plus en plus nombreux à avoir réalisé des stages, occupé des " petits boulots ", exercé des emplois saisonniers ou même plus réguliers. La moitié des étudiants exerce ainsi une activité rémunérée pendant son cursus. Avec l'expansion des stages obligatoires, leur nombre ne peut que s'accroître. Tout en prenant acte de cette évolution, l'auteur souligne la variété des stages et de leur contenu, qui influence la qualité de l'insertion professionnelle. De même que la définition d'un stage est "floue " (p. 123), la durée des stages, les conditions d'apprentissage et de suivi des stagiaires, etc., sont particulièrement diversifiées entre les diplômes, cursus, années de formation, départements universitaires, etc.

L'auteur montre ainsi qu'il est difficile de garantir les effets professionnels d'un stage, avant d'incriminer "l'insuffisante reconnaissance des compétences des étudiants» (p. 131).

Cette critique l'incite à regretter l'omission dont souffrent les compétences transversales acquises par les étudiants lorsqu'ils mènent des recherches documentaires, entreprennent des travaux d'enquête, rédigent des synthèses et des analyses, écrivent un mémoire...

On ne peut que déplorer avec lui cette vacance, entretenue par les enseignants, qui sousestiment la dimension professionnalisante d'exercices trop souvent jugés seulement " académiques ». L'inquiétude que soulève l'usage forcené du terme de "compétence » les conduit à s'en défier et ne pas considérer des activités qui sont bien de l'ordre du travail, même si ce dernier est universitaire.

Le cinquième chapitre interroge « les transformations des cursus de formation à l'aune de l'insertion professionnelle" (p. 137). L'auteur y décrit les principes de construction de l'offre de formation universitaire, tout à fait spécifiques et historiquement peu ouverts aux acteurs extérieurs à l'université, et met en valeur les évolutions qu'ils ont subies depuis les années 1980.

Désireux de convaincre les universitaires de s'engager dans la voie d'une professionnalisation de qualité, José Rose justifie certains changements qu'il considère comme liés aux évolutions plus générales de la société, et se livre à un exercice de prospective qui en souligne à son tour la nécessité.

Lorientation occupe une place importante dans ce chapitre, mais elle est abordée avec des éléments bien différents de ceux que mobilisent Sophie Orange et Romuald Bodin dans leur ouvrage sur la pseudo-crise des universités (2013) (L'université n'est pas en crise, Bellecombe en Bauges, Editions du Croquant, 2013). En se fondant sur plusieurs enquêtes nationales et locales, ces deux auteurs montrent en effet que l'orientation dans certains cursus sélectifs ne relève pas de choix raisonnés et que les taux d'échec en médecine et en 
droit devraient interroger les responsables politiques bien plus que ceux, bien moindres, d'autres cursus. L'inscription en BTS (brevet de technicien supérieur) résulte, par exemple, plus souvent d'une non-décision que d'un véritable choix, incité par les enseignants et par une " raison pratique " peu à l'écoute des désirs des jeunes.

Si José Rose met en cause de nombreux lieux communs sur les modes d'accès à l'emploi et sur les relations entre système éducatif et système productif, il se montre moins disposé à le faire lorsqu'il s'agit d'évoquer l'orientation, alors qu'elle représente un objet permanent d'interrogations et de débats, non sans raison.

L'ouvrage se termine sur le pilotage de la mission d'aide à l'insertion et sur les diverses activités qui relèvent d'une telle aide.

L'auteur se situe explicitement dans la prescription, afin de permettre aux universitaires de dépasser leurs craintes à l'égard de ces nouvelles activités et de réduire les clivages qu'elles suscitent dans leur milieu professionnel.

Il s'agit pour lui " d'améliorer la qualité des services rendus et l'efficacité des établissements d'enseignement supérieur» (p. 187). Il propose de mettre en place différents partenariats, de "construire un service spécialisé et multiplier les initiatives" (p. 189) et présente l'expérience de quelques dispositifs : le projet Elite et son diplôme universitaire d'aide à l'insertion, mis en place à l'université de Provence ; le réseau des utilisateurs du Portefeuille d'expériences et de compétences (PEC), qui implique désormais une trentaine d'universités.

Il aborde ensuite les méthodes de suivi et d'évaluation des dispositifs existants, dont les BAIP, en revenant sur les résultats déjà accumulés et en en déduisant quelques leçons susceptibles d'inspirer les démarches d'évaluation à venir. L'approche est à la fois pratique, questionneuse et prospective ; il s'agit de montrer, comme l'annonce le titre de l'ouvrage, que le défi lancé par la mise en place d'une mission d'insertion à l'université est à la fois nécessaire et tenable.

Fort de très nombreuses informations, résultats d'enquêtes, lectures mais aussi résultats d'expérimentations auxquelles l'auteur a directement contribué, cet ouvrage représente une somme pour tous ceux qui s'intéressent à l'insertion, à ses missions et à leurs outils.

Les responsables politiques, les enseignants-chercheurs et les étudiants y trouveront des réponses à leurs multiples interrogations, ainsi que maintes propositions. La présentation de nombreux paragraphes sous forme de liste a pour objectif de les équiper, même s'il est possible que la lecture en soit parfois rendue un peu difficile. Fins connaisseurs de ces questions ou débutants, ils y puiseront d'innombrables ressources pour mieux penser l'insertion des étudiants et les équiper en conséquence.

Ils n'y trouveront pas, en revanche, tous les termes des débats que suscite l'inscription de l'insertion parmi les missions des universités et des universitaires. José Rose a choisi de valoriser cette mission d'insertion et à cet effet, il privilégie ses dimensions positives, 
même s'il n'hésite pas à souligner les manques, obstacles, problèmes qui accompagnent l'inscription durable d'un tel dispositif au sein des universités.

Aspirant au changement dans les pratiques universitaires, il se veut résolument optimiste. Cette posture est peu fréquente dans le milieu universitaire et elle l'est encore moins depuis que la réforme permanente est devenue la règle. On doit en remercier l'auteur, même lorsqu'on ne partage pas sa conviction et encore moins son optimisme, car il invite à considérer autrement ce qu'on observe d'un œil toujours circonspect, et ouvre largement un débat qui se réduit trop souvent à une critique expéditive.

- Référence de l'ouvrage

Rose J. (2014), Mission insertion. Un défi pour les universités, Rennes : PUR, 237 p.

- Bibliographie

Orange S. et Bodin R. (2013), L'université n'est pas en crise, Bellecombe en Bauges, Editions du Croquant. 